\title{
SASH1 inhibits cervical cancer cell proliferation and invasion by suppressing the FAK pathway
}

\author{
HUI CHEN, DONGLIANG WANG and YULING LIU \\ Department of Gynecology and Obstetrics, The Second Affiliated Hospital of Zhengzhou University, \\ Zhengzhou, Henan 450014, P.R. China
}

Received January 21, 2015; Accepted November 6, 2015

DOI: $10.3892 / \mathrm{mmr} .2016 .4946$

\begin{abstract}
SAM and SH3 domain containing 1 (SASH1), a member of the SLY-family of signal adapter proteins, is a candidate tumor suppressor in several types of cancer. However, the role of SASH1 in cervical cancer remains to be elucidated. Therefore, in the present study, the role of SASH1 in cervical cancer and the underlying mechanism was investigated. Cell proliferation was detected by the MTT assay. Cell invasion was measured by Transwell assay. The mRNA expression levels of SASH1, matrix metalloproteinase (MMP)-2 and MMP-9 were determined by reverse transcription quantitative polymerase chain reaction. The protein expression levels of SASH1, MMP-2, MMP-9 and focal adhesion kinase (FAK) were determined by western blot analysis. The results demonstrated that SASH1 was downregulated in cervical cancer tissues and cell lines. Subsequently, a vector that overexpresses SASH1 was constructed. Overexpression of SASH1 was found to significantly inhibit cervical cancer cell proliferation and invasion, and also significantly reduce the expression of MMP-2 and MMP-9 in cancer cells. In addition, SASH1 modulated the FAK signaling pathway. Overexpression of SASH1 suppressed the expression of FAK in cancer cells. Taken together, the results suggested that SASH1 inhibits cervical cancer cell proliferation and invasion by suppressing the FAK pathway.
\end{abstract}

\section{Introduction}

Cervical cancer is the third most common type of malignant tumor and the fourth leading cause of cancer mortality among females worldwide (1). In developing countries, 500,000 females develop cervical cancer and $\sim 270,000$ females succumb to this disease, every year (2). Although chemotherapy and radiotherapy remain the major

Correspondence to: Dr Yuling Liu, Department of Gynecology and Obstetrics, The Second Affiliated Hospital of Zhengzhou University, 2 Jingba Street, Zhengzhou, Henan 450014, P.R. China

E-mail: yulingliusec@163.com

Key words: SAM and SH3 domain containing 1, cervical cancer, proliferation, invasion treatments for invasive cervical cancer, the five-year survival rate is limited due to the limited efficacy and high toxicity of numerous anticancer drugs. Therefore, further studies on the mechanisms of cervical cancer and the identification of new and effective gene therapy targets are important for the development of new treatment strategies and the improvement of patient survival.

The SAM and SH3 domain containing 1 (SASH1) gene, a member of the SLY-family of signal adapter proteins, encodes a protein containing sterile $\alpha$ motif (SAM) and Src homology domain 3 (SH3), predominantly observed in signaling molecules, adapters and scaffold proteins (3). SAM domains can interact with other protein domains (4) and SH3 domains bind to proline-rich motifs in proteins (5). These two domains are frequently found in signal adapter proteins and scaffolding factors. In previous years, numerous studies have revealed that SASH1 is downregulated in various types of tumor. Meng et al reported that the expression of SASH1 in osteosarcoma tissue was significantly lower than that in normal bone tissue and that SASH1 significantly reduced osteosarcoma cell viability, proliferation and invasive ability compared with the empty vector group and blank control group (6). In addition, the expression of SASH1 was lower in colon cancer compared with the levels found in normal colon tissue (7). However, the role of SASH1 in cervical carcinogenesis remains to be elucidated. Therefore, in the present study, the role of SASH1 in cervical cancer was investigated. The present findings demonstrated that SASH1 was downregulated in cervical cancer and that SASH1 inhibited the proliferation and invasion of cervical cancer cells by suppressing the focal adhesion kinase (FAK) pathway, indicating that SASH1 may be a potential therapeutic target in cervical cancer.

\section{Materials and methods}

Human tissue samples. A total of 30 normal cervical and 17 cervical cancer samples without chemotherapy, immunotherapy or radiotherapy were obtained via surgery from The Second Affiliated Hospital of the Medical College of Zhengzhou University (Zhengzhou, China) between January 2012 and December 2013. The present study was approved by the Ethics Committee of the Medical College of Zhengzhou University and informed consent was obtained from the patients prior to sample collection. 
Cell lines and cell culture. Human cervical cancer cell lines (SiHa, C33A and CaSki) and the normal colonic epithelial cell line (CRL-1831) were purchased from the American Type Culture Collection (ATCC, Rockville, MD, USA). All cell lines were cultured in RPMI-1640 medium (HyClone, Logan, UT, USA) supplemented with $10 \%$ fetal bovine serum (HyClone), $100 \mathrm{U} / \mathrm{ml}$ penicillin and $100 \mu \mathrm{g} / \mathrm{ml}$ streptomycin (Sigma-Aldrich, St. Louis, MO, USA) and grown at $37^{\circ} \mathrm{C}$ in an incubator with $5 \% \mathrm{CO}_{2}$. The medium was replaced once every 2-3 days and cells were digested with $0.25 \%$ trypsin and passaged when they reached $80 \%$ confluence.

Plasmid construction and stable transfection. The full-length coding sequence of the human SASH1 gene was amplified by reverse transcription quantitative polymerase chain reaction (RT-qPCR) and ligated into the pGEM-T vector (Clontech Laboratories, Inc., Cambridge, UK) at the EcoRI and $K p n I$ restriction sites. Following sequence confirmation, positive clones were subcloned into the pcDNA3.1 expression vector to construct the recombinant expression vector pcDNA3.1-SASH1.

For in vitro transfection, the cells were seeded in six-well plates at a density of $1.0 \times 10^{5}$ cells/well and cultured overnight. When the cells grew to $70-80 \%$ confluence, pcDNA3.1-SASH1 and the empty vector were transfected using Lipofectamine 2000 ${ }^{\mathrm{TM}}$ (Invitrogen; Thermo Fisher Scientific, Inc., Waltham, MA, USA) according to the manufacturer's instructions.

$R T-q P C R$. RNA was extracted from macrodissected primary tumor tissues or cultured cells homogenized in TRIzol reagent according to the manufacturer's instructions (Invitrogen; Thermo Fisher Scientific, Inc.). The Superscript First-Strand kit was used to synthesize first strand cDNA (Invitrogen; Thermo Fisher Scientific, Inc.). RT-qPCR was performed using an ABI Prism 7500 Sequence Detection System (Applied Biosystems, Foster City, CA, USA) and the following primers were used: SASH1, sense 5'-CGGGAA AGCGTCAAGTCGGA-3' and antisense 5'-ATCTCCTTT CTTGAGCTTGAG-3'; matrix metalloproteinase (MMP)-2, sense 5'-CCCCAGACAGGTGATCTTGAC-3' and antisense 5'-GCT TGCGAGGGA AGA AGT TG-3'; MMP-9, sense 5'-CGCTGGGCTTAGATCATTCC-3' and antisense 5'-AGG TTGGATACATCACTGCATTAGG-3'. The levels of individual gene mRNA transcripts were initially normalized to the control $\beta$-actin. Subsequently, the differential expression of these genes was analyzed using the $2^{-\Delta \Delta \mathrm{Cq}}$ method.

Western blot analysis. Equal quantities $(30 \mu \mathrm{g})$ of protein were solubilized in Laemmli buffer $(62.5 \mathrm{mM}$ Tris $/ \mathrm{HCl}$ pH 6.8, 10\% glycerol, 2\% SDS, 5\% mercaptoethanol and $0.00625 \%$ bromophenol blue), boiled for $5 \mathrm{~min}$, separated by $12 \%$ polyacrylamide gel electrophoresis and transferred onto nitrocellulose membranes (EMD Millipore, Bedford, MA, USA). The membranes were probed with rabbit anti-SASH1 (cat. no. LS-C285219; LifeSpan Biosciences, Seattle, WA, USA), rabbit anti-MMP-2 (cat. no. 4022S), rabbit anti-MMP-9 (cat. no. 3852S), rabbit anti-FAK (cat. no. 3285S) or mouse anti- $\beta$-actin (cat.no. 4967S) (all from Cell Signaling Technology Inc., Beverly, MA, USA) in Tris-buffered saline with $0.1 \%$
Tween 20 (TBST) containing 5\% BSA (Sigma-Aldrich) at $4{ }^{\circ} \mathrm{C}$ overnight, followed by three washes in TBST for 5 min per wash. The membranes were incubated with horseradish peroxidase-conjugated goat anti-rabbit IgG polyclonal antibody (cat. no. MBS560261; Mybiosource, San Diego, CA, USA) for $1 \mathrm{~h}$ at room temperature. The blotted protein bands were exposed to and visualized using enhanced chemiluminescence (ECL) reagent. Developed films were digitized by scanning, and the optical densities were analyzed using Image J software 1.61 (National Institutes of Health, Bethesda, MD, USA).

Cell proliferation assay. Cell viability was determined using an MTT assay as described previously (8). In brief, cells were plated in 96 -well culture plates at $5 \times 10^{3}$ cells per well and incubated overnight. Cells were treated with pcDNA3.1-SASH1 vector or empty vector for 24, 48, 72 and $96 \mathrm{~h}$. Following this, MTT was added to each well. The resulting formazan was then dissolved in $100 \mu \mathrm{l}$ of dimethyl sulfoxide and the absorbance was recorded at $540 \mathrm{~nm}$ using a Bio-Rad 3350 microplate reader (Bio-Rad Laboratories, Hercules, CA, USA).

Colony formation assay. Cells were seeded into 10-mm dishes at a density of $2 \times 10^{2}$ cells/dish and then cultured at $37^{\circ} \mathrm{C}$ for 10-14 days until the visible cell clones were formed. Following this, the cells were fixed with methanol. Fixative buffer was discarded and the cells were stained with Giemsa dye for $10 \mathrm{~min}$. After the staining solution had been discarded, the number of cell clones was counted.

Cell invasion assay. Cell invasion was measured using a modified Boyden chamber (BD Biosciences, Bedford, MA, USA). Cells were treated with overexpression-SASH1 or the mock for $4 \mathrm{~h}$, and were then seeded in the upper chamber. DMEM medium with $10 \%$ fetal bovine serum was added into the lower chamber. After $24 \mathrm{~h}$ of incubation, cells that passed through the lower side of the membrane were stained with hematoxylin and eosin (Sigma-Aldrich) and quantified using a BX51 fluorescence microscope (Olympus Optical Co., Tokyo, Japan) by counting six high-powered fields in the center of each well.

Statistical analysis. Statistical analysis was performed using SPSS software version 16.0 (SPSS, Inc., Chicago, IL, USA). For comparison among groups, a $\chi^{2}$ test or a one-way analysis of variance followed by a post hoc Tukey's test was performed. $\mathrm{P}<0.05$ was considered to indicate a statistically significant difference. The data are presented as the mean \pm standard deviation.

\section{Results}

Expression of SASH1 is decreased in cervical cancer tissues and cells. To examine the role of SASH1 in cervical cancer tumorigenesis, RT-qPCR analyses of cervical cancer tissues and normal cervical tissues was performed. The results demonstrated that the mRNA expression of SASH1 was markedly lower in cervical cancer tissues compared with the normal cervical tissues (Fig. 1A). Similar results were obtained from the analysis of cervical cancer cell lines. As shown in Fig. 1B and C, the mRNA and protein expression levels of SASH1 were also significantly lower in cervical 
$\mathbf{A}$

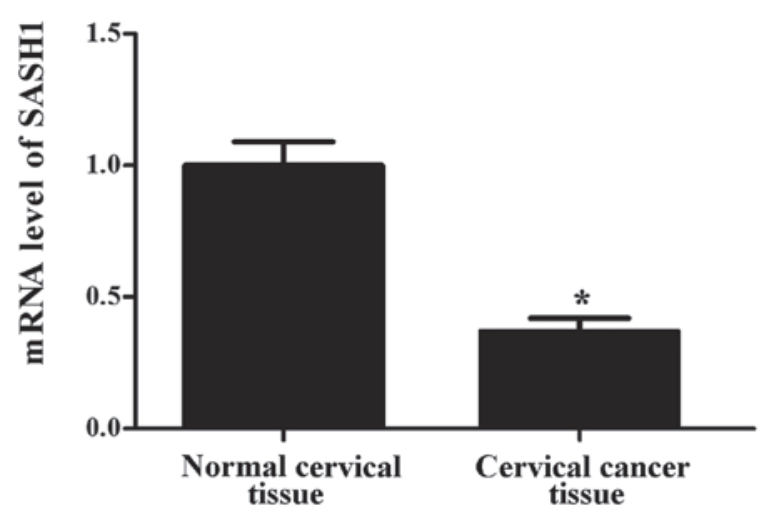

B

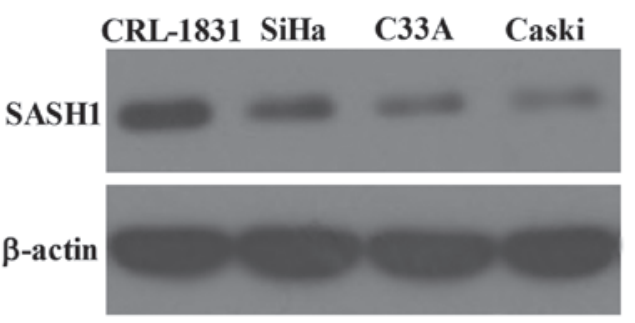

C

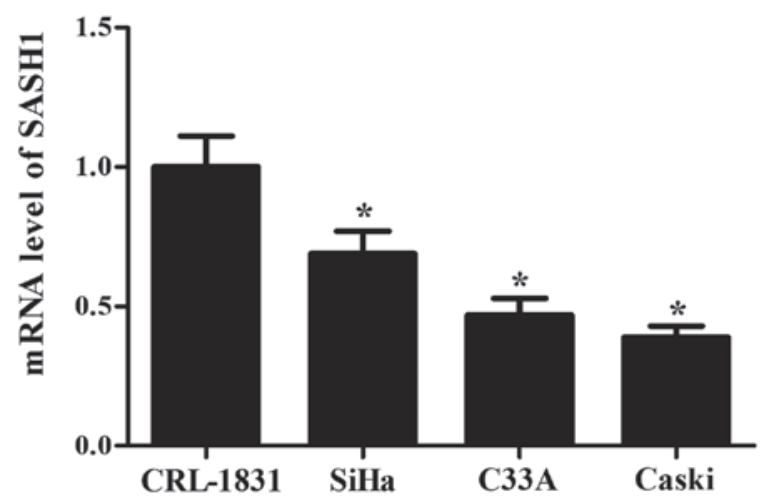

Figure 1. SASH1 expression in cervical cancer tissues and cell lines. (A) RT-qPCR analysis of SASH1 expression in normal cervical and cervical cancer tissues. (B) Expression of SASH1 in SiHa, C33A and CaSki cells was measured by western blot analysis. (C) Expression of SASH1 in SiHa, C33A and CaSki cells was measured by RT-qPCR. Values are presented as the mean \pm standard deviation. ${ }^{*}<<0.05$. SASH 1, SAM and SH3 domain containing 1; RT-qPCR, reverse transcription-polymerase chain reaction.

cancer cell lines compared with the CRL-1831 group. Collectively, these data suggest that SASH1 is downregulated in cervical cancer.

Expression of SASH1 is increased following transfection with $p c D N A 3.1-S A S H 1$. To further investigate the effect of SASH1 on cervical cancer tumorigenesis, the recombinant expression vector pcDNA3.1-SASH1 was constructed, and then RT-qPCR and western blot analysis were employed to determine the efficiency of SASH1 transfection. As shown in Fig. 2A, the mRNA expression of SASH1 was significantly higher in the pcDNA3.1-SASH1-transfected group relative to the control group. No significant difference in the mRNA expression level of SASH1 between the control group and the empty vector group was identified. Consistent with the results of RT-qPCR, western blot analysis demonstrated
A

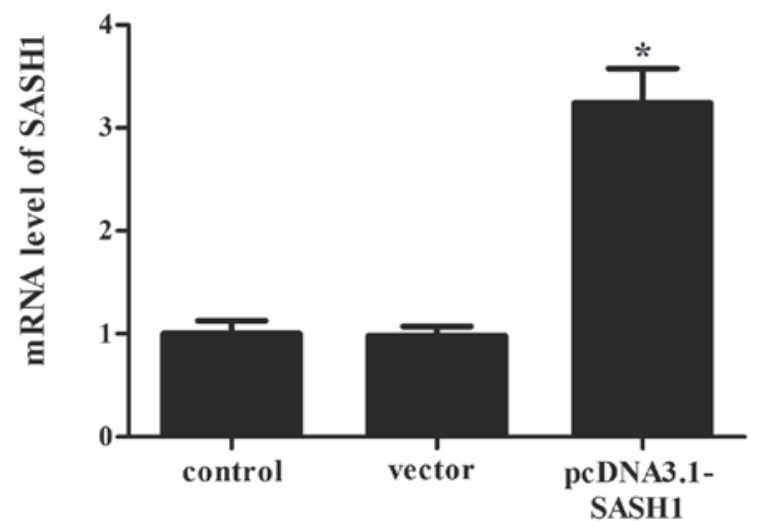

B

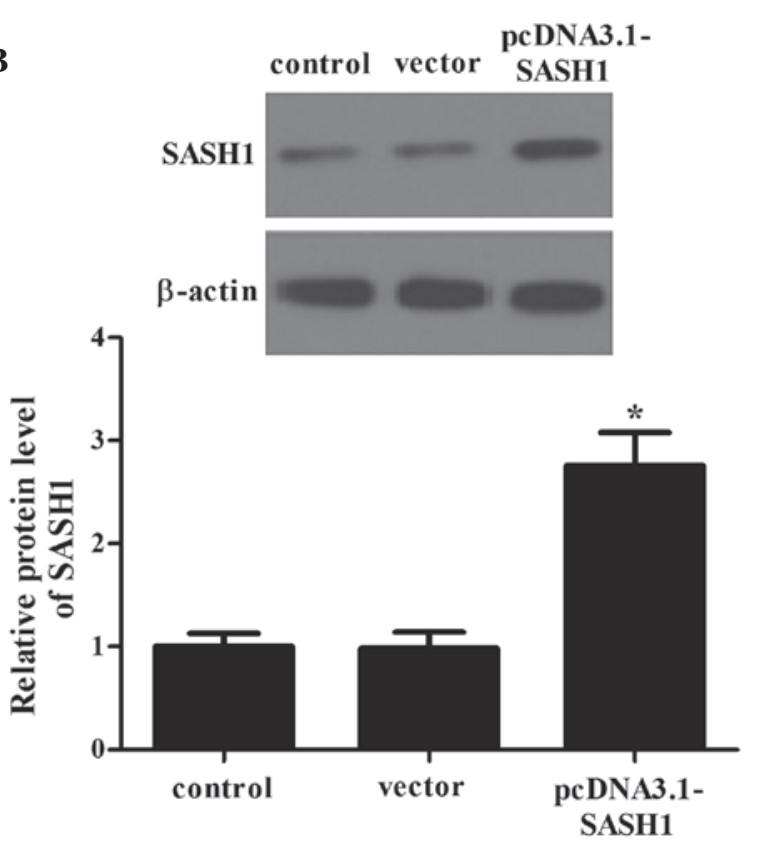

Figure 2. Determination of transfection effects. (A) Representative images of the relative mRNA levels of SASH1 in cells transfected with the empty vector and pcDNA3.1-SASH1. (B) Representative western blots of control, empty vector and pcDNA3.1-SASH1-transfected cells. The expression levels of proteins were normalized based on $\beta$-actin levels. All experiments were repeated at least three times and all data are presented as the mean \pm standard deviation. ${ }^{*} \mathrm{P}<0.05$, compared with the control or vector group. SASH1, SAM and SH3 domain containing 1.

that the expression of SASH1 protein was also significantly increased in the pcDNA3.1-SASH1-transfected group (Fig. 2B). Collectively, these data show that the expression of SASH1 was successfully elevated in the CaSki cell line.

SASH1 inhibits cervical cancer cell proliferation. Subsequently, the effect of SASH1 on cervical cancer cell proliferation was investigated. As shown in Fig. 3A, SASH1 significantly inhibited the proliferation of cervical cancer cells, in a time-independent manner. In addition, the effect of SASH1 on colony formation in soft agar was investigated. SASH1 also reduced the colony formation in soft agar relative to the control (lentiviral vector)-transduced cells (Fig. 3B). These data indicate that SASH1 inhibits cervical cancer cell proliferation. 


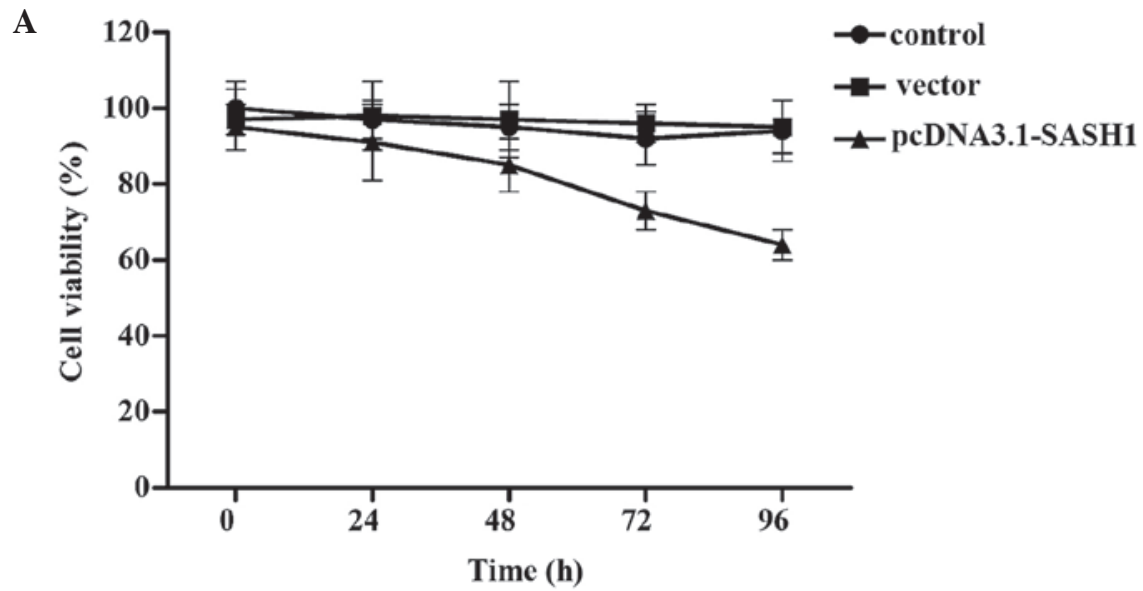

B
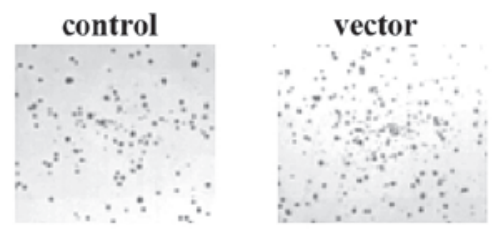

pcDNA3.1-SASH1

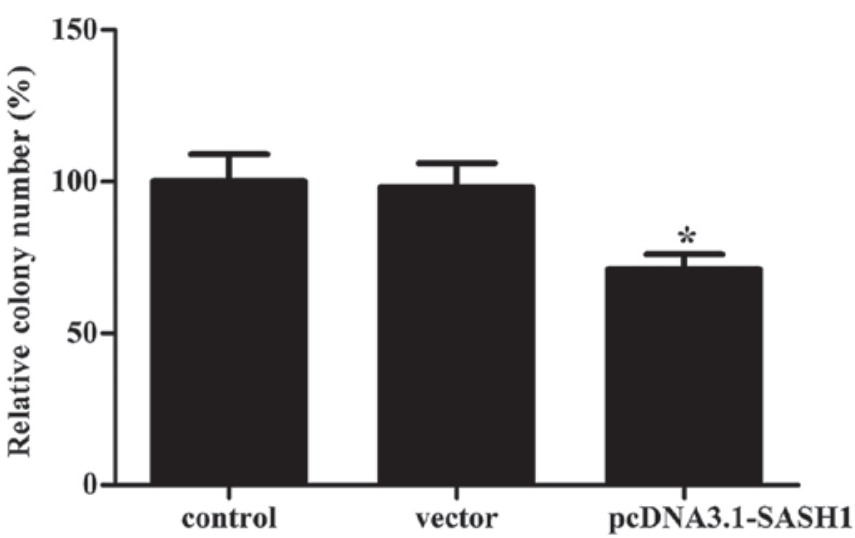

Figure 3. Effect of SASH1 on the proliferation of cervical cancer cells in vitro. (A) SASH1 inhibited the proliferative activity of cervical cancer cells. (B) Relative colony number in control cells, cells stably transfected with the empty pcDNA3 vector and cells stably transfected with the pcDNA3.1-SASH1 expression vector. All experiments were repeated at least three times and all data are presented as the mean \pm standard deviation. ${ }^{*} \mathrm{P}<0.05$, compared with the control or empty vector group. SASH1, SAM and SH3 domain containing 1.

SASH1 inhibits cervical cancer cell invasion. The invasive behavior of cervical cancer cells was determined using a Boyden chamber assay. Cervical cancer cells demonstrated a reduced capacity for invasion when transfected with pcDNA3.1-SASH1 (Fig. 4A). Furthermore, since the upregulation of MMP-2 and MMP-9 expression contributes to the invasiveness of cancer cells $(9,10)$, the effects of SASH1 on MMP-2 and MMP-9 was investigated in cervical cancer cells. As shown in Fig. 4B and C, SASH1 significantly decreased the expression levels of MMP-2 and MMP-9.

SASH1 inhibits cervical cancer cell proliferation and invasion through the FAK pathway. The FAK pathway is involved in cell proliferation and invasion. The present study thus examined whether SASH1 affects the level of FAK. As shown in Fig. 5, SASH1 overexpression significantly inhibited the expression level of FAK. This indicated that SASH1 may be involved in the regulation of the FAK pathway as an upstream molecule, suggesting that SASH1 regulates cell proliferation and invasion via the FAK pathway.

\section{Discussion}

Previous studies have demonstrated that SASH1 is important in tumorigenesis and tumor development. However, the role of SASH1 in cervical cancer remains to be elucidated. The present study demonstrated that SASH1 expression was downregulated in cervical cancer tissues and cell lines. SASH1 overexpression inhibited cell proliferation and invasion. Furthermore, SASH1 inhibited cervical cancer cell proliferation and invasion through the FAK pathway.

Several studies have demonstrated that SASH1 is downregulated in tumors and that this expression is correlated with tumor grade and prognosis. For example, in breast cancer cell lines, SASH1 is only expressed at low levels and is downregulated in the majority (74\%) of breast tumors compared with corresponding normal breast epithelial tissues (3). The expression of SASH1 is also downregulated in tumors of the lung (11). In addition, the expression levels of SASH1 were significantly reduced in colon cancer at Union for International Cancer Control stage II, III and IV, as well as in liver metastases, and 
A

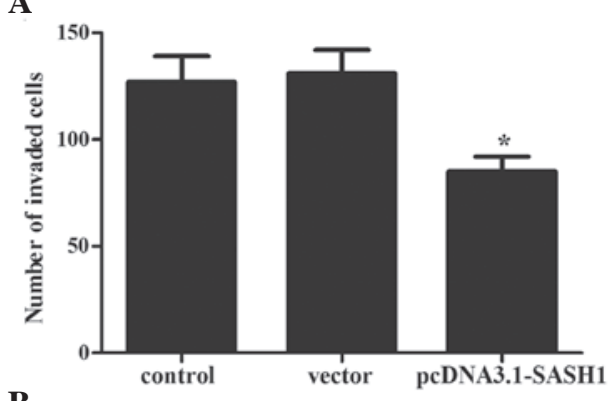

$\mathbf{B}$

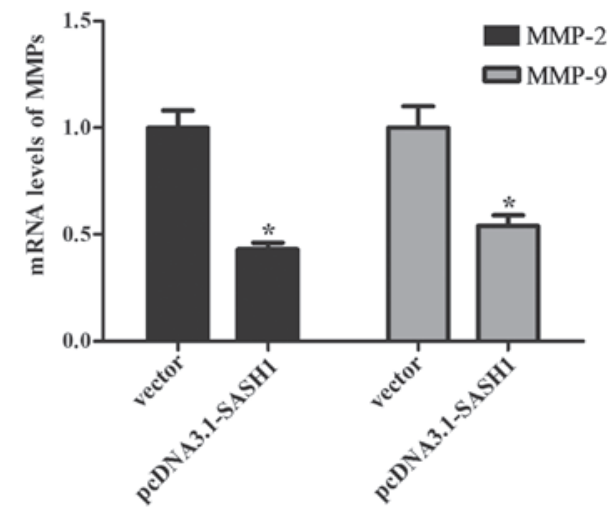

C

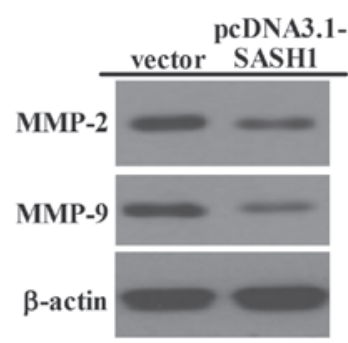

Figure 4. Effect of SASH1 on the invasion of cervical cancer cells in vitro (A) The number of invaded cells was significantly decreased following pcDNA3.1-SASH1 transfection. (B) mRNA levels of MMP-2 and MMP-9 examined by western blot analysis. (C) Protein levels of MMP-2 and MMP-9 examined by western blot analysis. ${ }^{*} \mathrm{P}<0.05$, compared with the empty vector group. SASH1, SAM and SH3 domain containing 1; MMP, matrix metalloproteinase.

the expression of SASH1 in colon tumors was lower compared with the levels found in normal colon tissue (7). Consistent with the abovementioned results, in the present study, SASH1 expression was examined in clinical tissue samples and cell lines, and it was found that SASH1 mRNA and protein expression was decreased in tumor tissues, suggesting that SASH1 may be a candidate tumor suppressor gene in cervical cancer.

The migration and invasion of cancer cells involves a host of processes and the interaction of multiple genes (12-14). MMPs are a major group of enzymes that regulate cellular matrix composition, and are zinc- and calcium-dependent endopeptidases. Among the MMPs, MMP-2 and MMP-9 have a pivotal role in the degradation of laminin, type IV collagen and gelatin, components of the extracellular matrix (ECM) and basement membrane (15). In addition, high levels of MMP-2 and MMP-9 expression have been demonstrated to be associated with cancer progression, invasion and metastasis (16). Therefore, inhibiting MMP-9 expression may be critical for treating malignant tumors. The present study found that
A
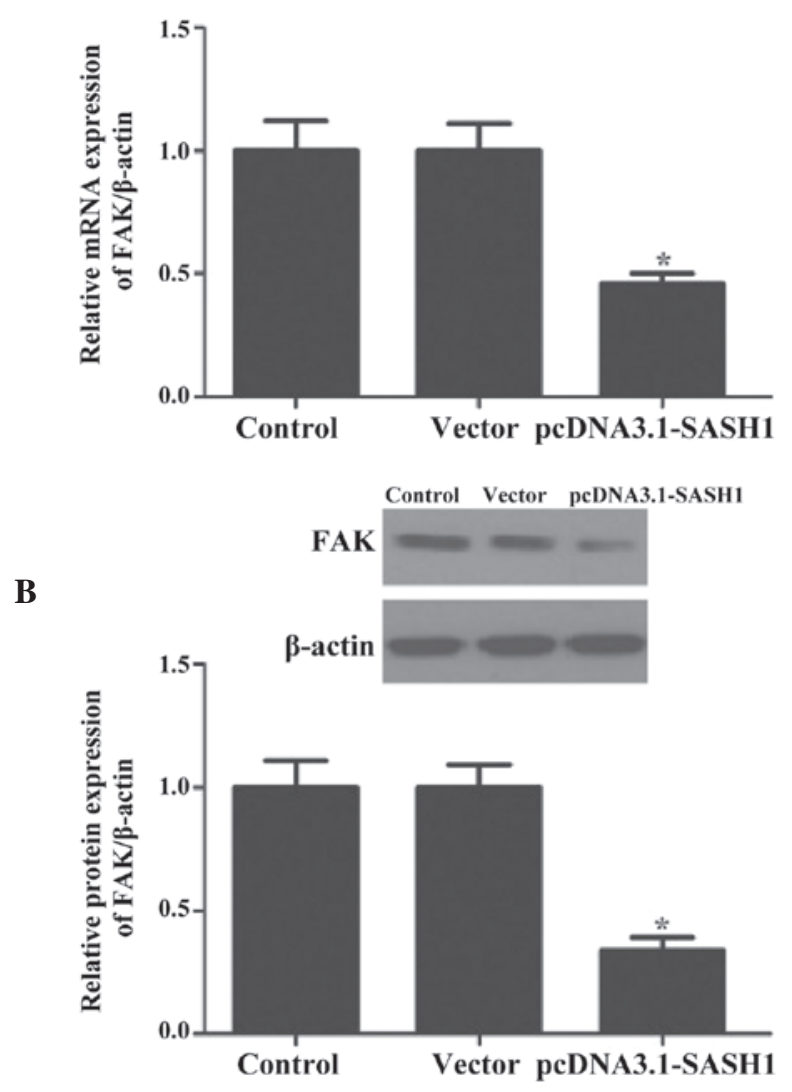

Figure 5. SASH1 inhibits cervical cancer cell proliferation and invasion through the FAK pathway. (A) SASH1 overexpression significantly inhibited the mRNA expression level of FAK. (B) SASH1 overexpression significantly inhibited the protein expression level of FAK. ${ }^{*} \mathrm{P}<0.05$, compared with the control or empty vector group. SASH1, SAM and SH3 domain containing 1; FAK, focal adhesion protein.

SASH1 inhibits the levels of MMP-2 and MMP-9 in cervical cancer cells, resulting in a reduction in invasive ability.

FAK, an intracellulartyrosine kinase, localizes at focal adhesions and is a major regulator of signals from the ECM. It can also mediate the signaling of various growth factor receptors, including epidermal growth factor receptor, platelet-derived growth factor receptor, G-protein coupled receptors and the hepatocyte growth factor receptor c-MET (17). It is involved in the regulation of cancer cell metastasis and survival and is associated with aggressive tumor behavior (18-20). Certain studies have demonstrated a direct role of FAK in promoting tumor growth. Breast tumors of higher histological grades and of a triple-negative sub-type express elevated levels of FAK (21). A small molecule FAK inhibitor (PF-228) prevented the migration of melanoma cells and the invasion of the highly invasive 1205Lu and WM9 melanoma cell lines (22). Inhibition of FAK signaling has been demonstrated to suppress the proliferation of Hep3 human carcinoma cells and leads to tumor dormancy in vivo, and this dormancy can be reversed by the expression of an active mutant of MEK1 (MAPK/ERK kinase 1) (23). The present study found that SASH1 overexpression reduced the level of FAK. This indicated that SASH1 could be an upstream regulator of FAK. These data strongly support the role of SASH1 in regulating cell migration and invasion via the FAK pathway. 
In conclusion, the present study demonstrated that SASH1 inhibits cervical cancer cell proliferation and invasion. Therefore, SASH1 may be important in cervical cancer and may represent a novel therapeutic target for cervical cancer treatment.

\section{References}

1. Greenlee RT, Murray T, Bolden S and Wingo PA: Cancer statistics, 2000. CA Cancer J Clin 50: 7-33, 2000.

2. Saavedra KP, Brebi PM and Roa JC: Epigenetic alterations in preneoplastic and neoplastic lesions of the cervix. Clin Epigenetics 4: 13, 2012.

3.Zeller C, Hinzmann B, Seitz S, Prokoph H, Burkhard-Goettges E, Fischer J, Jandrig B, Schwarz LE, Rosenthal A and Scherneck S: SASH1: A candidate tumor suppressor gene on chromosome $6 \mathrm{q} 24.3$ is downregulated in breast cancer. Oncogene 22: 2972-2983, 2003.

4. Kim CA, Gingery M, Pilpa RM and Bowie JU: The SAM domain of polyhomeotic forms a helical polymer. Nat Struct Biol 9: 453-457, 2002.

5. Pawson T: Protein modules and signalling networks. Nature 373: 573-580, 1995.

6. Meng Q, Zheng M, Liu H, Song C, Zhang W, Yan J, Qin L and Liu X: SASH1 regulates proliferation, apoptosis, and invasion of osteosarcoma cell. Mol Cell Biochem 373: 201-210, 2013.

7. Rimkus C, Martini M, Friederichs J, Rosenberg R, Doll D, Siewert JR, Holzmann B and Janssen KP: Prognostic significance of downregulated expression of the candidate tumour suppressor gene SASH1 in colon cancer. Brit J Cancer 95: 1419-1423, 2006.

8. Wu GS, Song YL, Yin ZQ, Guo JJ, Wang SP, Zhao WW, Chen XP, Zhang QW, Lu JJ and Wang YT: Ganoderiol A-enriched extract suppresses migration and adhesion of MDA-MB-231 cells by inhibiting FAK-SRC-paxillin cascade pathway. PLoS One 8: e76620, 2013.

9. Chambers AF and Matrisian LM: Changing views of the role of matrix metalloproteinases in metastasis. J Natl Cancer Inst 89: 1260-1270, 1997.

10. McCawley LJ and Matrisian LM: Matrix metalloproteinases: Multifunctional contributors to tumor progression. Mol Med Today 6: 149-156, 2000.

11. Chen EG, Chen Y, Dong LL and Zhang JS: Effects of SASH1 on lung cancer cell proliferation, apoptosis, and invasion in vitro. Tumor Biol 33: 1393-1401, 2012.
12. Beshir AB, Ren G, Magpusao AN, Barone LM, Yeung KC and Fenteany G: Raf kinase inhibitor protein suppresses nuclear factor- $\kappa \mathrm{B}$-dependent cancer cell invasion through negative regulation of matrix metalloproteinase expression. Cancer Lett 299: $137-149,2010$

13. Wang Z, Li Y, Banerjee S, Kong D, Ahmad A, Nogueira V, Hay N and Sarkar FH: Down-regulation of Notch-1 and Jagged-1 inhibits prostate cancer cell growth, migration and invasion and induces apoptosis via inactivation of Akt, mTOR, and NF-kappaB signaling pathways. J Cell Biochem 109: 726-736, 2010.

14. Arozarena I, Sanchez-Laorden B, Packer L, Hidalgo-Carcedo C, Hayward R, Viros A, Sahai Eand Marais R: Oncogenic BRAFinduces melanoma cell invasion by downregulating the cGMP-specific phosphodiesterase PDE5A. Cancer Cell 19: 45-57, 2011.

15. Lai WC, Zhou M, Shankavaram U, Peng G and Wahl LM: Differential regulation of lipopolysaccharide-induced monocyte matrix metalloproteinase (MMP)-1 and MMP-9 by p38 and extracellular signal-regulated kinase 1/2 mitogen-activated protein kinases. J Immunol 170: 6244-6249, 2003.

16. Bauvois B: New facets of matrix metalloproteinases MMP-2 and MMP-9 as cell surface transducers: Outside-in signaling and relationship to tumor progression. Biochim Biophys Acta 1825: 29-36, 2012.

17. Luo M and Guan JL: Focal adhesion kinase: A prominent determinant in breast cancer initiation, progression and metastasis Cancer Lett 289: 127-139, 2010.

18. Lark AL, Livasy CA, Calvo B, Caskey L, Moore DT, Yang X and Cance WG: Overexpression of focal adhesion kinase in primary colorectal carcinomas and colorectal liver metastases: Immunohistochemistry and real-time PCR analyses. Clin Cancer Res 9: 215-222, 2003.

19. Zhao J and Guan JL: Signal transduction by focal adhesion kinase in cancer. Cancer Metastasis Rev 28: 35-49, 2009.

20. Ward KK, Tancioni I, Lawson C, Miller NL, Jean C, Chen XL, Uryu S, Kim J, Tarin D, Stupack DG, et al: Inhibition of focal adhesion kinase (FAK) activity prevents anchorage-independent ovarian carcinoma cell growth and tumor progression. Clin Exp Metastasis 30: 579-594, 2013.

21. Yom CK, Noh DY, Kim WH and Kim HS: Clinical significance of high focal adhesion kinase gene copy number and overexpression in invasive breast cancer. Breast Cancer Res Treat 128: 647-655, 2011.

22. John JK, Paraiso KH, Rebecca VW, Cantini LP, Abel EV, Pagano N, Meggers E, Mathew R, Krepler C, Izumi V, et al: GSK3 $\beta$ inhibition blocks melanoma cell/host interactions by downregulating $\mathrm{N}$-cadherin expression and decreasing FAK phosphorylation. J Invest Dermatol 132: 2818-2827, 2012.

23. Aguirre Ghiso JA: Inhibition of FAK signaling activated by urokinase receptor induces dormancy in human carcinoma cells in vivo. Oncogene 21: 2513-2524, 2002. 